\title{
Morbidity and Mortality from Measles in Cameroonian Children: Implications for Measles Control
}

\author{
Félicitée Nguefack*,1, Mathurin Tejiokem ${ }^{2}$, Andreas Chiabi*,1, Roger Dongmo ${ }^{3}$, Innocent Kago ${ }^{1}$, \\ Tetanye Ekoe ${ }^{1}$, Boubacar Dieng ${ }^{4}$ and Innocent Takougang ${ }^{5}$
}

\author{
${ }^{\text {I} D e p a r t m e n t ~ o f ~ P a e d i a t r i c s, ~ F a c u l t y ~ o f ~ M e d i c i n e ~ a n d ~ B i o m e d i c a l ~ S c i e n c e s, ~ U n i v e r s i t y ~ o f ~ Y a o u n d e ~ I, ~ P . O . ~ B o x ~ 1364, ~}$ \\ Yaounde, Cameroon \\ ${ }^{2}$ Centre Pasteur of Cameroon, Member of the International Network of Pasteur Institutes, PO Box 1274 Yaoundé, \\ Cameroon \\ ${ }^{3}$ Gynaeco-Obstetrical service, Efoulan District Hospital, Cameroun \\ ${ }^{4}$ United Nations Children's Fund (UNICEF), P.O. Box 1181 Yaoundé, Cameroon \\ ${ }^{5}$ Department of Public Health, Faculty of Medicine and Biomedical Sciences University of Yaounde I, Cameroon
}

\begin{abstract}
Objective: To investigate the epidemiological trends of measles in the Littoral, North-west, South-west and Western regions of Cameroon in order to improve measles control.

Design and Methods: Cross-sectional study of retrolective data collected on cases of measles from January 1997 to April 2003 and stored in registers at regional levels. Child age, immunization and disease status, complications and outcomes were recorded.

Results: 33,268 cases of measles were registered. The trend was endemo-epidemic with outbreaks occurring between February and April. The average incidence rate was 4.8\%o per year with 4.1\%o reported in $1998,5.8 \%$ in 2001 , and $0.9 \%$ in 2002. Complete data sets for 16,637 cases indicate that $47.4 \%$ were children aged 9 to 59 months. Those less than 9 months represented $15.2 \%$ of the cases. More than half ( 415 cases) of the 766 cases with known immunization status $(54.2 \%)$ representing $2.5 \%$ of the 16,637 total cases had received the measles vaccine. Complications were frequent in the Western $(53.7 \%)$ and the Littoral $(33.7 \%)$ regions and $53.3 \%$ of all complications were bronchopulmonary infections. The global case fatality rate was $1.6 \%$, and $2.1 \%$ in children less than 9 months with a significant tendency to decrease with age $\left(\mathrm{p}<10^{-4}\right)$. The most frequent cause-related disorders of death were malnutrition $(34.4 \%)$, neurological $(35.3 \%)$ and digestive $(26.2 \%)$.

Conclusion: measles was a public health problem in Cameroon with vaccinated and unvaccinated children both affected within the period of study. Those less than 9 months old, carried the highest burden of the disease in terms of mortality.
\end{abstract}

Keywords: Measles, morbidity, mortality, vaccination, Cameroon.

\section{INTRODUCTION}

Measles is a cosmopolitan infectious disease with high morbidity and mortality rates. It is influenced by family size, population density, birth rate, population movement and immunization coverage [1]. Despite widespread availability of the measles vaccine, about 39.9 million cases occurred in 2000 , and killed some 614,000 children worldwide [2,3]. Until recently, epidemics were registered each 3-4 years, particularly in developing countries where children who had received vaccine shots were also affected [4-6]. Low coverage, primary vaccination failure $[7,8]$, and poor maintenance

*Address correspondence to these authors at the Department of Paediatrics, Faculty of Medicine and Biomedical Sciences, University of Yaounde I, Cameroon, P.O. Box 1364, Yaounde, Cameroon; Tel: 00 (237) 995914 08, 00 (237): 995983 71;

E-mails: dongfel@yahoo.fr, andy_chiabi@yahoo.co.uk of the cold chain are the main reasons given to explain these outbreaks. Waning of measles vaccine-induced immunity can lead to the occurrence of measles in immunized children [9]. The duration of immunity induced by primary vaccination is known to be long lasting [10].

Measles is a disease with a proven safe and cost-effective intervention. Its eradication is feasible [11] with high vaccination coverage among infants through routine health services. Proper handling of vaccines is mandatory for effective measles control.

Since 1986, the implementation of the one-dose, measles vaccine was provided to children at 9 months in Cameroon, using the facility-based or out-reaches mobile strategy. However coverage was less than $50 \%$ for a decade. Up to 2001, Cameroon was one of the 20 countries of the world, with frequent measles epidemics. In the three northern regions, epidemics occurred yearly or every three years in the 
seven other regions of Cameroon [12]. Supplemental immunization activities (SIAs) leads to high vaccination coverage and more measles cases and deaths averted, compared to the single dose schedule $[13,14]$. Since the implementation of the SIAs in Cameroon, the incidence of measles has declined $[15,16]$.

Before 2000, measles surveillance in Cameroon was poor on aggregate numbers of cases reported by health facilities. Parameters such as age-specific rates, vaccination status, complications and outcome following measles were not well documented, especially in the southern part of Cameroon (Littoral, North-west, South-West, and Western regions), where frequent epidemics were noted. The aim of this study was to assess the epidemiological trend of measles in this part of Cameroon to have baseline information on control program operations.

\section{MATERIALS AND METHODOLOGY}

\section{Study Design and Data Collection}

This cross-sectional study was carried out over six months, from October 2002 to April 2003 and reviewed data on measles cases over a period of six years four months
(January 1997 to April 2003). This study focused on two steps of measles surveillance in Cameroon: the passive and the active phases. During the active phase, measles suspected cases were investigated and the blood tested for the presence of IgM antibodies against the measles virus. Four regions of Cameroon (Littoral, North West, South West, and West) were studied (Fig. 1). These regions were selected because of their demographic weight, high measles- related morbidity and low vaccination coverage and frequent measles epidemics. The study consisted of systematically reviewing the disease report forms at the level of the district and regional public health services. The investigator (F.N.) collected data with the support of the local health personnel at the health facilities and the administrative services. Registers in the health facilities where the measles cases were reported were next reviewed.

Data was recorded using the weekly, the monthly and yearly report forms for reportable diseases of the Cameroon's Ministry of Public Health, and was obtained from the district health services and the regional delegations of public health. Missing data at this level was obtained from registers in health facilities.

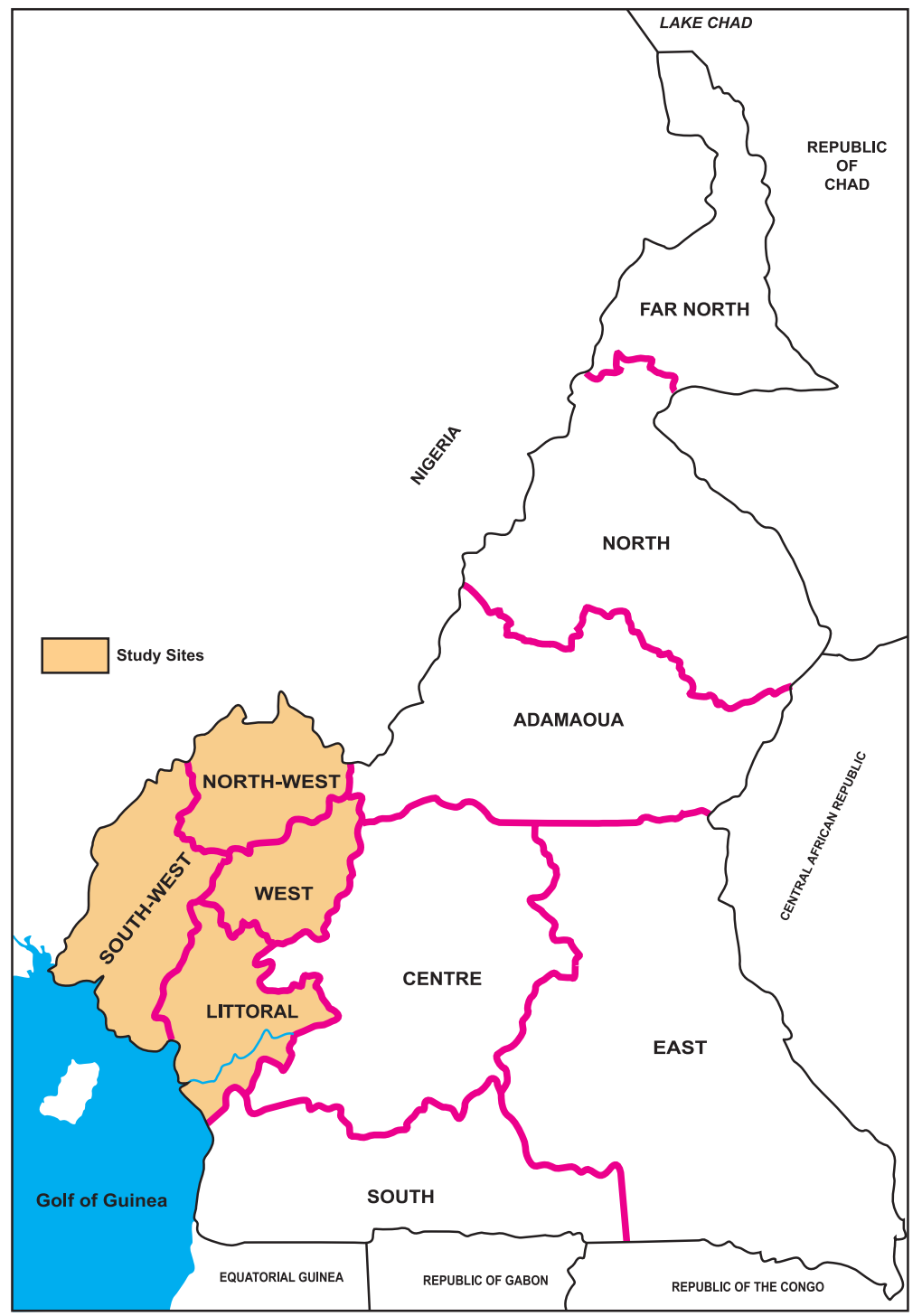

Fig. (1). Map of Cameroon showing the study sites. 


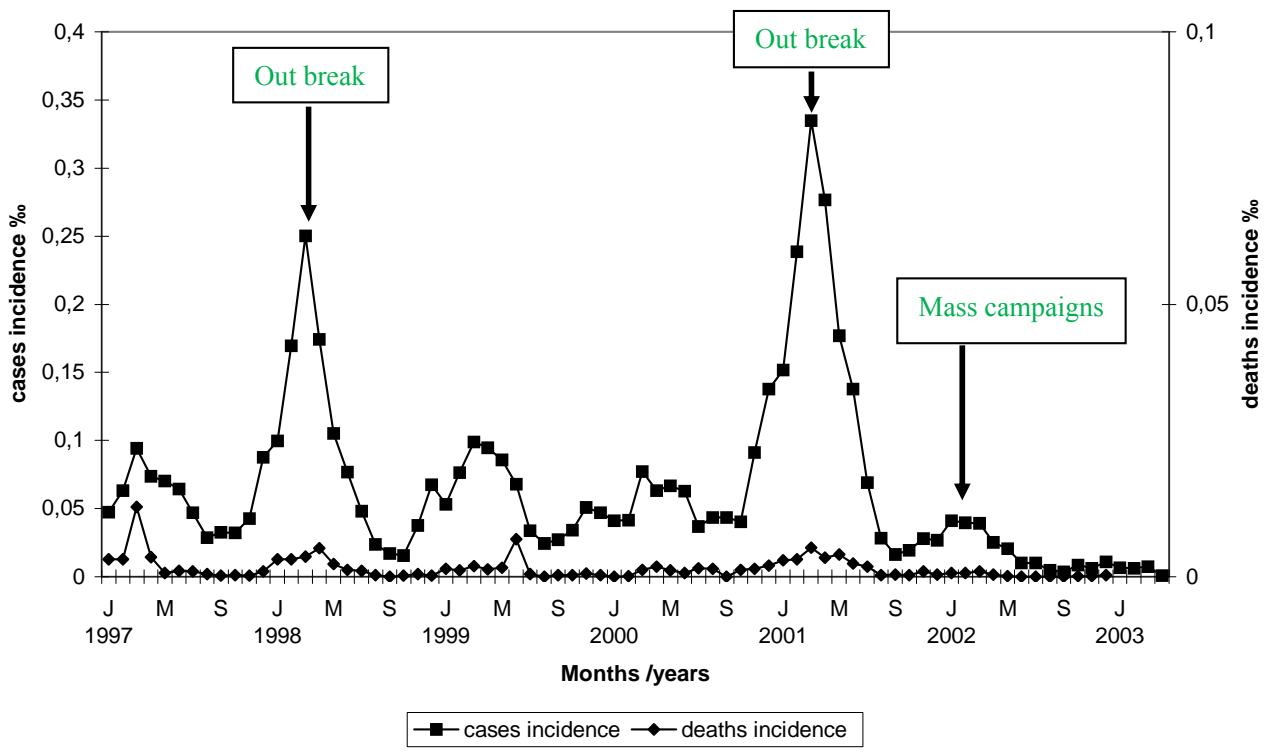

Fig. (2). Cumulative incidence of measles in the Littoral, North-West, South-West and Western regions of Cameroon (January 1997 - April 2003).

Computations were performed with reference to the demographic data of the 1987 official census population which was 10.5 million inhabitants.

This study was approved by the ethics committee of the Cameroon's Ministry of Public Health.

\section{Data Collection on the Parameters of Measles Cases}

It was based on the inpatient and outpatient registers of the health facilities.

The clinical case definition of measles in this study was the presence of fever and a generalized maculopapular rash, and any of the following symptoms (coryza, cough, or conjunctivitis) according to WHO [17]. Only the case definition was used until September 2002.
Since October 2002, any case that meets the case definition of measles is investigated in Cameroon. Blood analyses are performed for measles IgM antibodies titers in "Centre Pasteur" of Cameroon. From the report forms for reportable diseases, cases recorded at the district health services were serologically followed up. Child age, sex, immunization status, place of residence, date of diagnosis, complications, treatment modalities, duration of hospitalization and outcome of the cases were recorded. The sample was comprised of all consecutive cases reported during the study period.

\section{Statistical Analysis}

The associations between variables were performed with the Chi square test. An association was significant if the $p$ value was $<0.05$.

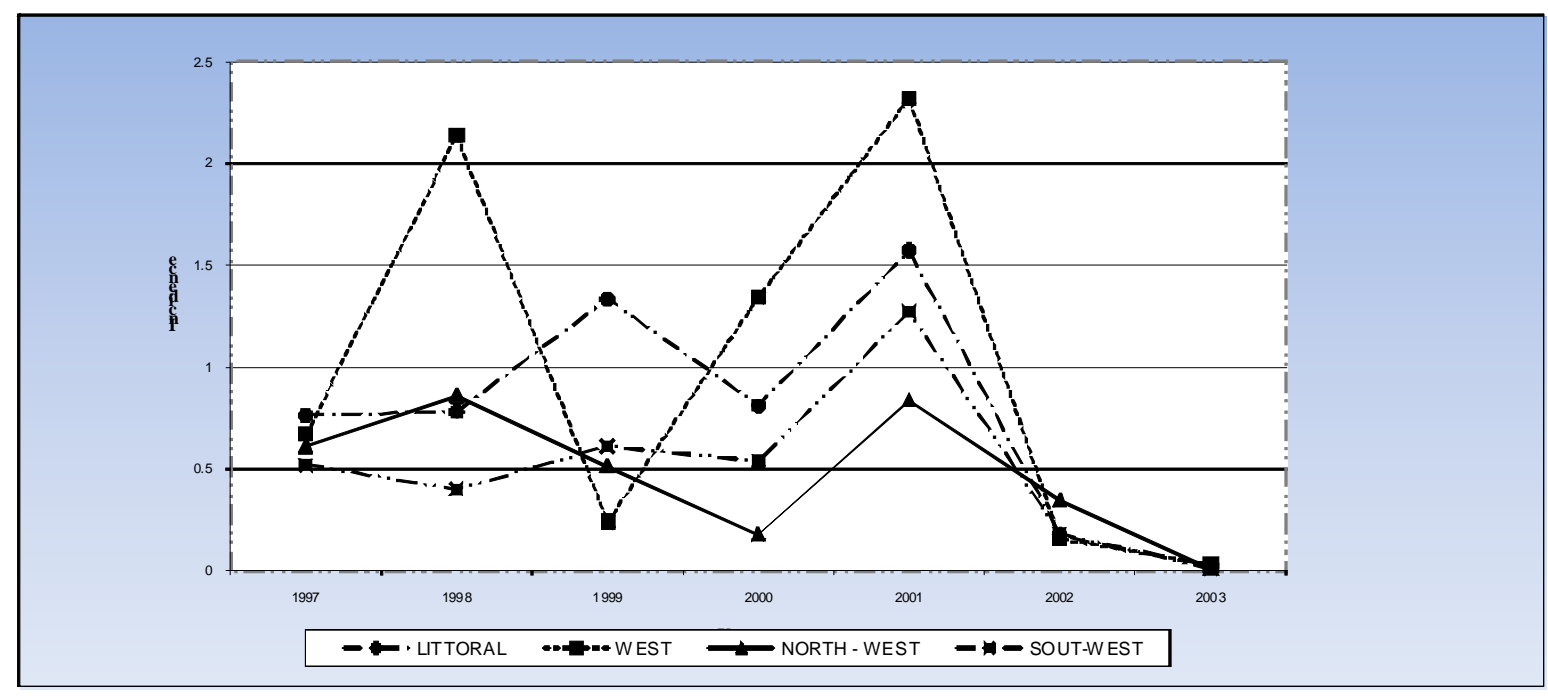

Fig. (3). Incidence of measles in the Littoral, North-West, Western and South-West regions (January 1997 - April 2003) has been improved so that the years on the abscissa could be seen easily. 


\section{RESULTS}

Measles Incidence from January 1997 to April 2003 and Distribution of Cases with Respect to Regions (Figs. 2 and 3)

In all, 33,268 cases were recorded from the 63 health districts of the four regions studied. Measles was endemoepidemic, as it occurred the whole year with outbreaks coinciding with the months of February to April (Fig. 2). The average incidence rates was $4.8 \%$ per year from January 1997 to December 2002, with outbreaks reported in 1998 (4.1\%o) and three years after (5.8 \%o in 2001). The lowest rate $(0.9 \%)$ occurred in 2002 . The Western region registered $36.2 \%$ of the cases, followed by Littoral (31.7\%), North-west $(19.9 \%)$ and South-west (12.7\%).

\section{Distribution of Cases with Age}

Complete data sets for 16,637 cases of public $(59 \%)$ and mission $(39.8 \%$ ) health facilities indicate that $47.4 \%$ were children aged 9 to 59 months. Those 60 months or above, and those less than 9 months represented $37.4 \%$ and $15.2 \%$ of the cases respectively (Fig. 4).

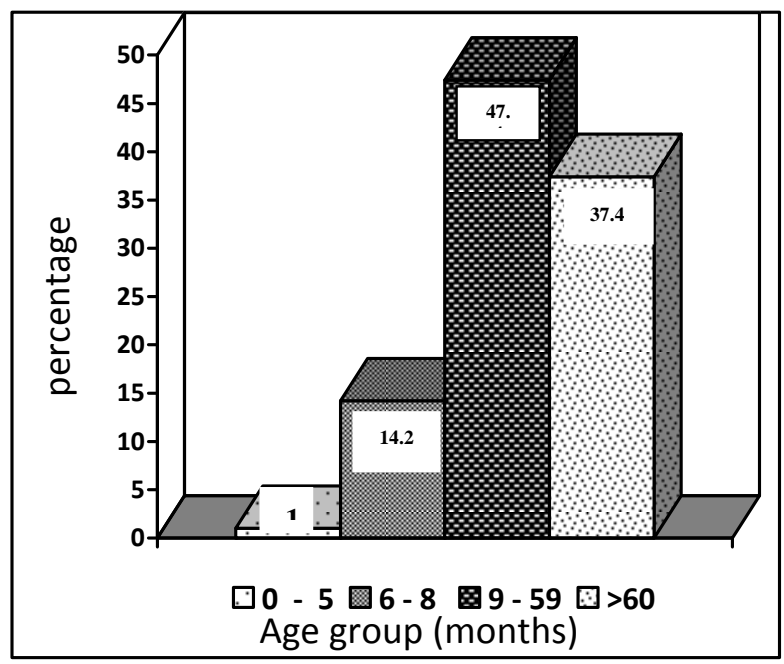

Fig. (4). Distribution of measles cases with age.

\section{Distribution of Measles Cases with Age and Reported Immunization Status}

The immunization status was not always mentioned in the registers in the health facilities. It was recorded in 766 (4.6\%) out of the total 16,637 cases of measles cases (Fig. 5). Among them, more than half (415 cases) representing $2.5 \%$ of the 16,637 cases had already been vaccinated against measles. The 0 to 9 months age group represented $15.3 \%$ of the measles cases.

In all, 273 out of $351(77.8 \%)$ of the unvaccinated cases were more than 9 months old. Fifty four percent of vaccinated cases were recorded in the 9 to 59 months age group.

\section{Distribution of Complications with Respect to Regions and Age}

Complications were frequent in the Western $(53.7 \%)$ and the Littoral (33.7\%) regions and 53.3\% were bronchopul- monary infections. Complications were equally distributed in the age groups, and $75.7 \%$ occurred in the less than 60 months' old, amongst which the less than 9 months represented $27.3 \%$.

\section{Disease Outcome}

The global case fatality rate was $1.6 \%$ (Table 1 ). The mortality rate was $2.7 \%$ in the South-west, $2.4 \%$ in the Western, $1.6 \%$ in the North-west and $0.6 \%$ in the Littoral regions. It was $2.1 \%$ and $2.2 \%$ respectively in children less than 9 months and in those of 9 to 12 months. Thereafter it decreased significantly with age $\left(\chi^{2}\right.$ for trend $\left.=43,8 \mathrm{p}<10^{-4}\right)$.

The most frequent cause-related disorders of death were malnutrition (34.4\%), neurological (35.3\%) and digestive $(26.2 \%)$. Most deaths occurred within the first 2 days following admission in the hospital.

\section{Distribution of Measles Cases According to Region and Serologic Tests (October 2002-April 2003)}

A total of 201 cases of measles were recorded during the first seven months (October 2002-April 2003) corresponding to the period which Cameroon started active measles surveillance phase. In the Littoral region, 38 occurred as an outbreak. Twelve out of 15 cases in which antibody titers were determined revealed the presence of anti-measles IgM $(80 \%)$ antibodies. Measles was confirmed in 4 of $5(80 \%)$ cases aged less than 6 months old and in half of the children less than 9 months.

\section{DISCUSSION}

Measles Incidence and Distribution of Cases with Respect to Regions

Measles was seasonal at the study sites, and its transmission was annual with outbreaks occurring between February and April, coinciding with the dry seasons. Outbreaks were reported in 1998 and three years after (in 2001). This pattern has also been described by Fauveau et al. [18]. The transmission rate of $0.9 \%$ observed in 2002 corresponded to the postepidemic periods and the intensification of the measles control periods, during which the vaccination coverage was reinforced in Cameroon.

The number of measles cases was elevated in the Western and the Littoral. These regions are amongst those with the highest population density. The population density was more than 1001 inhabitants per $\mathrm{km}^{2}$ in the Littoral; the three other regions had equal population densities (more than 250 inhabitants per $\mathrm{km}^{2}$ ). This parameter alone could not explain the rate of occurrence of measles. Population movement, vaccination coverage and climatic conditions could have influenced the trend of the disease in these regions.

\section{Distribution of Cases with Age}

Measles occurred mostly in children aged less than 59 months in the four regions of the study. It affected $15 \%$ of children less than 9 months old, the age recommended for vaccination against measles in Cameroon. During an outbreak in 2001 in Yaounde, $92 \%$ of measles cases tested revealed the presence of IgM antibodies [6]. At the same time, the virus was isolated in $81 \%$ of the cases, and the 12 to 59 


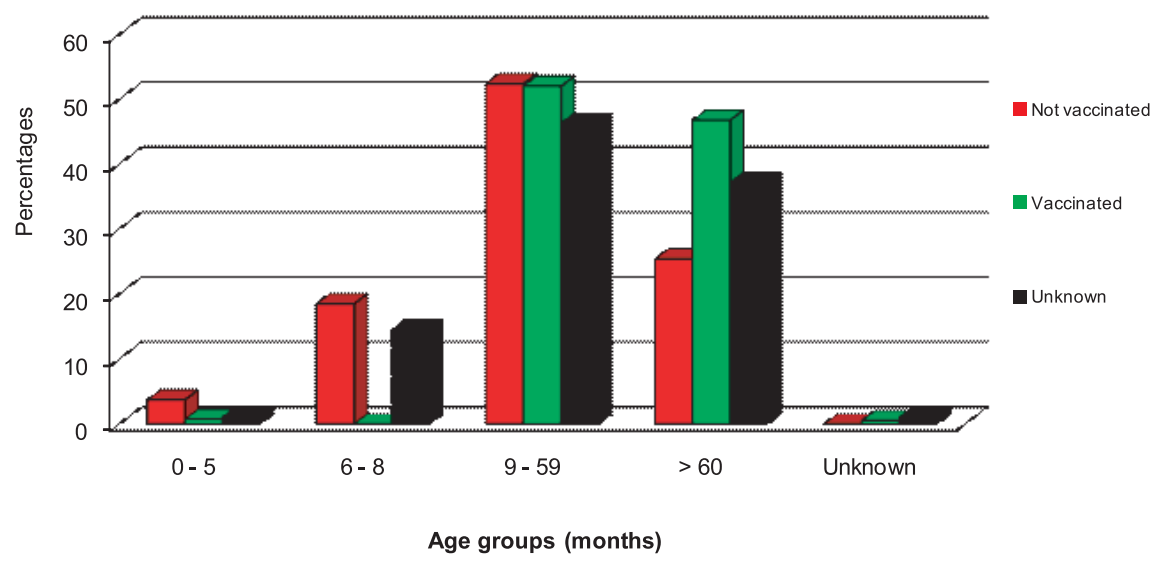

Fig. (5). Distribution of the cases of measles according to age and vaccination status.

Table 1. Outcome of the Measles Cases with Age

\begin{tabular}{|c|c|c|c|c|}
\hline Age Groups (Months) & Total Cases N (\%) & Lost to Follow up N (\%) & Recovered N (\%) & Dead N (\%) \\
\hline \hline $0-5$ & $163(1.1)$ & $15(9.2)$ & $143(87.7)$ & $5(3.1)$ \\
\hline $6-8$ & $2125(14.2)$ & $470(22.2)$ & $5647(79.4)$ & $44(2.0)$ \\
\hline $9-59$ & $7110(47.4)$ & $1300(18.3)$ & $4503(80.3)$ & $163(2.3)$ \\
\hline$\geq 60$ & $5604(37.3)$ & $1069(19.1)$ & $11904(79.3)$ & $244(1.6)$ \\
\hline
\end{tabular}

months old children represented $62 \%$ of cases [6]. Even in rural Bangladesh [18], children aged less than 9 months old suffered the greatest burden of this disease although they were not eligible for vaccination. Kiepiela et al. demonstrated that maternally acquired measles antibody titres rapidly declined so that at 9 months, children are no longer protected [19]. Considering the expanded program of immunization (EPI), the question now is should the anti-measles vaccine be implemented earlier? According to Nokles et al. [20], it is not beneficial to simply reduce the vaccination age. A two doses of measles vaccination policy is more efficient for measles control than the one dose currently administered $[13,21-23,28-30]$, but budgetary constraints constitute a serious barrier for the implementation of this strategy. Besides, a multidose program may distract the delivery of the routine vaccine at 9 months to the most vulnerable children [24].

\section{Distribution of Measles Cases with Age and Reported Immunization Status}

Measles affected both vaccinated and unvaccinated children in Cameroon. Concerning measles amongst vaccinated children, similar situations have been reported in others settings $[6,7,25]$. The persistence of maternally acquired measles antibodies at the age of vaccination could have also induced vaccine failure. For this reason, even with good vaccine conditions, $15 \%$ of children, failed to be protected [26]. The ideal is to push the age of measles vaccine delivery at 12 to 15 months but, children will no longer have maternally acquired antibodies and many will develop measles before the vaccination. In developed countries, measles affected vaccinated and older children, with the $5-9$ years age group representing $1 / 3$ of the cases [27]. In the highest vaccination coverage settings, it occurred mostly among vaccinated children during epidemics [28]. The situation is different in our context where the vaccination coverage remained low, and where the most affected age group were less than 60 months old.

Primary failure and waning of vaccine-induced immunity cannot explain our results. Waning immunity is not a risk factor of measles [29], but rather the effectiveness of the measles vaccine. In Cameroon, vaccines may be delivered under suboptimal conditions, and this might have increased the susceptibility of infants to measles. Poor quality of the cold chain in the vaccine storage, transportation, handling and ineffective immunization technique could be some of reasons for failure. Some health facilities in Cameroon do not have refrigerators and power failure occurs and may last for many days. We also noted that in some health facilities, in the refrigerators used to store vaccines, drinking water and foodstuffs were also stored alongside.

In the unvaccinated children, low vaccination coverage as a risk factor of the occurrence of measles [24] is not due to the vaccine shortage in our context. In fact, $90 \%$ of health facilities had a continuous supply of vaccine [30], but all the children were not targeted. Cultural barriers combined with low socioeconomic status and the inadequate quality of health care delivery could limit the population from access to the vaccine.

\section{Disease Outcome}

Bronchopulmonary infections were the most frequent complications (53.3\%) followed by neurogical (convulsions) and digestives (mostly diarrhoea). Some studies revealed 
that, two-thirds of measles cases developed respiratory tract complications and diarrhea [31], and these disorders were most frequently associated with death [7,32,33]. According to Kabir et al, failure to vaccinate children in the first year of life against measles contributes to $27 \%$ increase in the mortality rate [34]. In the present study, patients were given antibiotics systematically and this practice could have reduced the number of bacterial infections which could be fatal. The case fatality rate $(1.6 \%)$ in this study is a problem, especially in children aged less than 9-months old. All deaths might not have been recorded because $41.2 \%$ of the measles cases were managed on outpatient basis and $17.4 \%$ were lost to followup.

\section{Limits of the Study}

The study was limited to the administrative health services and the health facilities. The under reporting and mishandling of data, the behavioural under-utilisation of health facilities for measles care could have rendered our data less reliable. One is tempted to ask whether the cases clinically diagnosed were really measles cases? This question can not be answered precisely since the biological proof of measles started later in 2002. Besides, the immunization status was not always noted and the vaccination records were not always seen. The study was also not limited to children and the number of inhabitants was derived from the 1987 population census. There might have been some bias related to the population at risk of measles.

\section{CONCLUSION}

Unless mass immunization campaigns are undertaken, measles remains a public health problem in the Littoral, North-West, South-West and Western regions of Cameroon, where epidemics were frequent. Measles was seasonal at the study sites with transmission annually, and outbreaks occurring after three years. Vaccinated and unvaccinated children were both affected. The most affected age group was the 959 months. In those less than 9 months old (not eligible for vaccination according to the EPI in Cameroon), mortality was high. Poor quality of the cold chain for vaccine storage, transportation, handling of the vaccines might have been some of the causes of the occurrence of measles in the vaccinated population.

We thus recommend that special attention should be given to the cold chain and reinforcement of the competence of health care workers in the proper handling of vaccines and best practices in vaccination techniques. The power managers (AES-SONEL) and health workers should be sensitised on the disastrous effect of the power shortages on vaccine storage and its consequences on the health of the population. The implementation of an integrated supervision of health services and health care activities needs to be improved. Finally, strategies to include a second vaccination opportunity and the improvement of the vaccination coverage are crucial for the reduction of the number of unvaccinated children and thus the occurrence of measles in the less than 9 months old.

To overcome re-occurrence of measles outbreaks, the Cameroon's Ministry of Public Health has adopted strategies based on the strengthening of the EPI, reinforcement of casebased surveillance of measles, and the implementation of mass immunization campaigns. Since 2002, the supplemen- tal immunization activities have enabled a drastic decrease in the incidence of measles in the country. The number of the confirmed cases decreased from 220 in 2002 to 41 cases in 2007 [34].

\section{ACKNOWLEDGEMENTS}

We thank the district medical officers, district health officers and my colleagues who work in the health facilities involved in this study, for their financial and material support. We thank also Drs Toh Ephraim and Soh Florence for helpful comments on the draft.

\section{REFERENCES}

[1] Walsh JA. Selective primary health care: strategies for control of disease in the developing world. IV. Measles Rev Infect Dis 1983; 5: $330-8$.

[2] Stein CE, BirminghamM, Kurian M, Duclos P, Strebel P. The Global Burden of Measles in the Year 2000-A Model that Uses Country-Specific indicators. J Infect Dis 2003; 1 87: S8-14.

[3] WHO/UNICEF. Joint statement: reducing measles mortality in emergencies. WHO/V\&B/04.03,UNICEF/PD/Measles/02.2004

[4] Godoy P, Dominguez A, Salleras L. Measles: effect of a two-dose vaccination programme in Catalonia, Spain. Bull World Health Organ 1999; 77: 132-37.

[5] Poland GA, Jacbson RM. Failure to reach the goal of measles elimination. Apparent paradox of measles infection in immunized persons. Arch Intern Med 1994; 154: 1815-20.

[6] Waku KD, Nerrienet E, Mfoupouendoun J, Tene G, Whittle H, Wild TF. Measles virus strains circulating in Central and West Africa: geographical distribution of two B3 genotypes. J Med Virol 2002; 68: 433- 44.

[7] Kambarami RA, Nathoo KJ, Nkrumah FK, Pirie DJ. Measles epidemic in Harare, Zimbabwe, despite high measles immunization coverage rates. Bull World Health Organ 1991: 69: 213-9.

[8] Güriş D, Bayazıt Y, Özdemirer Ü, et al. Measles epidemiology and elimination strategies in Turkey. J Infect Dis 2003; 187: S230-34.

[9] AabyP, Cisse B, Simondon F, Samb B, Soumare M, Whittle HP. Waning of vaccine-induced immunity: is it a problem in Africa? Am J Epidemiol 1999; 149: 304-05

[10] Bin D, Zhihui C, Qichang L, et al. Duration of immunity following immunization with live measles vaccine: 15 years of observation in Zhejiang Province, China. Bull World Health Organ1991; 69: 41523.

[11] Barett S. Eradication versus control: the economics of global infectious disease policies. Bull World Health Organ 2004; 89: 683-88.

[12] Cummings DA, Moss WJ, Long $\mathrm{K}$, et al. Improved measles surveillance in Cameroon reveals two major dynamic patterns of incidence. Int J Infect Dis 2006; 10: 148-55.

[13] Wolfson LJ, Strebel PM, Gacic-Dobo M, Hoekstra EJ, McFarland JW, Hersh BS. Has the 2005 measles mortality reduction goal been achieved? A natural history modelling study. Lancet 2007; 369: 191-200.

[14] Dayan GH, Cairns L, Sangrujee N, et al. Cost-effectiveness of three different vaccination strategies against measles in Zambian children. Vaccine 2004; 22: 475-84.

[15] Wiysonge CS, Nomo E, Ngo-Ndjan J, Ticha JM. Accelerated measles control in sub-Saharan Africa . Lancet 2006; 367: 394-5.

[16] Otten, M, Kezaala R, Fall A, et al. Public-health impact of accelerated measles control in the WHO African Region 2000-03. Lancet 2005; 366: 832-39.

[17] WHO-recommended standards for surveillance of selected vaccine-preventable diseases. Geneva: WHO 2003 (WHO/V\&B/ 03.01).

[18] Fauveau V, Chakraborty J, Sarder AM, Khan MA, Koenig MA. Measles among under-9-month-olds in rural Bangladesh: its significance for age at immunization. Bull World Health Organ 1991; 69: 67-72.

[19] Kiepiela P, Covadia HM, Loening WEK. Loss of maternal measles antibody in black South African infants in the first year of life: implications for age of vaccination. S Afr Med J 1991; 79: 145-48.

[20] Nokles DJ, Maclean AR, Anderson RM. Measles immunization strategies for countries with high transmission rates: Interim guide- 
lines predicted using a mathematical model. Int J Epidemiol 1990; 19: 703-10.

[21] Ota MO, Moss Mj, Griffin DE. Emerging diseases: measles. J Neurobiol 2005; 11: 447-54.

[22] Tulchinsky TH, Ginsberg GM, Abed Y, et al. Measles control in developing and developed countries: The case for a two-dose policy. Bull World Health Organ 1993; 71: 93-103.

[23] Garly ML, Martins CL, Balé C, et al. Early two-dose measles vaccination schedule in Guinea-Bissau: good protection and coverage in infancy. Int J Epidemiol 1999; 28: 347-52.

[24] Strebel p, Guris D, Papania M, Cochi S. Vaccine failure or failure to vaccinate? Am J Epidemiol 1999; 15: 295-301.

[25] Ceylan A, Ertem M, Korukluoðlu G, et al. An epidemic caused by measles virus type D6 in Turkey. Turk J Pediatr 2005; 47: 309-15.

[26] Cutts FT, Dabis F. Contrôle de la rougeole dans les pays en développement. Santé 1994; 4: 163-71.

[27] Chauvin P, Valleron AJ. Dix années de surveillance épidémiologique de la rougeole en France à travers un réseau de médecins sentinelles. Santé 1994; 4: 191-4.

[28] Soula G. Au sujet d'un paradoxe, ou comment répondre aux rumeurs parfois désagréables à propos des cas de rougeole survenant chez des sujets vaccinés. Bull Liais Doc OCEAC 1998; 31: 57-8.
[29] Hennessey KA, Ion-Nedelcu N, Craciun MD, et al. Measles Epidemic in Romania, 1996-1998: Assessment of Vaccine Effectiveness by Case-Control and Cohort Studies. Am J Epidemiol 1999; 150: $1250-7$.

[30] Waters HR, Dougherty L, Tegang SP, et al. Coverage and costs of childhood immunizations in Cameroon. Bull World Health Organ 2004; 82: 668-75

[31] Moss WJ. Measles still has a devastating impact in unvaccinated populations. PLoS Med 2007; 4: e24.

[32] Narain JP, Sharshi K, Rana SRS, Banerjee KB. Epidemic in an isolated unvaccinated population, India. Int J Epidemiol 1989; 18 : 952-57.

[33] Rodgers DV, Gindler JS, Atkinson WL, Markowitz LE. High attack rates and case fatality during a measles outbreak in groups with religious exemption to vaccination. Pediatr Infect Dis J 1993; 12: 288-92.

[34] Kabir Z, Long J, Reddaiah VPR, et al. Non specific measles vaccination on overall child mortality in an area of rural India with high vaccination coverage: a population-base-control study. Bull World Health Organ 2003; 81: 244-50.

[35] Department of Public Health. Complete Multi-Year Plan 2007 2011 of the Expanded Programme on Immunization 2008: 31

Received: February 04, 2011

Revised: April 01, 2011

Accepted: April 05, 2011

(C) Nguefack et al.; Licensee Bentham Open.

This is an open access article licensed under the terms of the Creative Commons Attribution Non-Commercial License (http://creativecommons.org/licenses/ by-nc/3.0/) which permits unrestricted, non-commercial use, distribution and reproduction in any medium, provided the work is properly cited. 